\title{
MARKETING DEPARTMENT'S INFLUENCE AND INFORMATION DISSEMINATION WITHIN IN A FIRM: EVIDENCE FOR AN INVERTED U-SHAPED RELATIONSHIP
}

\author{
Martin Schmidt, University of St. Gallen, Switzerland \\ Johannes Hattula, University of St. Gallen, Switzerland \\ Christian Schmitz, University of St. Gallen, Switzerland \\ Sven Reinecke, University of St. Gallen, Switzerland
}

\begin{abstract}
The dissemination of market intelligence across an organization is a key task of a firm's marketing department, as recognized by marketing researchers (e.g., Maltz and Kohli 1996) and practitioners (e.g., Mohr et al. 2010). They place great emphasis on market intelligence dissemination, a main component of firm's (internal) market orientation (e.g., Jaworski and Kohli 1993), due the strategic importance (Makadok and Barney 2001) and its positive effects on the financial performance of a firm (e.g., Kumar et al. 1998). In order to enhance intelligence dissemination throughout the firm, conventional wisdom in the market orientation literature suggests marketing department's influence within the firm as an important driver (Verhoef and Leeflang 2009). Hereby, past research (e.g., Moorman and Rust 1999; Verhoef et al. 2011) has generally assumed a positive linear relationship between marketing influence and market intelligence dissemination, and subsequently on performance. Our research, however, questions an entirely positive linear relationship. Specifically, building on social psychological research that implies that the degree of influence affects communication behavior (e.g., Keltner et al. 2003), we suggest that high marketing influence can ironically backfire. Hence, we propose that marketing influence has an inverted Ushaped relationship with market intelligence dissemination, which in turn impacts financial firm performance.
\end{abstract}

The findings of two studies, using survey and experimental data, support our prediction of a U-shaped relationship between marketing influence and market intelligence dissemination. First, cross-industrial data was obtained from 194 marketing managers from several European countries via an online survey. To measure marketing influence within the firm, market intelligence dissemination, and firms' financial performance, we used items from existing literature (cf. Moorman and Rust 1999; Luo et al. 2006; Kumar et al. 2011). To test our hypotheses, we included the quadratic term of marketing influence to examine the potential U-shaped relation in addition to the linear term. To analyze our model, we used the unconstrained model proposed by Marsh, Wen, and Hau (2006). As expected, we find a positive and significant linear effect of marketing influence on its intelligence dissemination $\left(\gamma_{11}=.164, p<.045\right)$, while the squared term has a significant negative effect $\left(\gamma_{11 \times 1}=-.181, p<.01\right)$. This supports the proposed inverted U-shaped relation. Moreover, the dissemination of intelligence has a positive effect on firms' financial performance $\left(\beta_{21}=.204, p<.01\right)$. Moreover, we designed a second study to test for the cause-effect relationship by manipulating marketing influence because of the possibility of reversed causality between marketing influence and intelligence dissemination (cf. Verhoef and Leeflang 2009). For study 2, we recruited 105 marketing managers to take part in an online study on strategic decision making. Participants were asked to place themselves in the role of head of marketing for a fictitious company and received a market report for the specific industry. Next, we manipulated the influence of the marketing department within the firm, whereby participants were randomly assigned to a high, a medium, or a low marketing influence condition. We then asked participants how likely it was that they would disseminate the market report to another department. The results of an ANOVA revealed a significant effect of the marketing influence manipulation $(\mathrm{F}=3.947, p<.022)$. Participants in the medium marketing influence condition $(\mathrm{M}=5.16)$ were significantly more likely to disseminate market intelligence than those in the low $(\mathrm{M}=4.59, \mathrm{~F}=6.196, p<.015)$ and high conditions $(\mathrm{M}=4.52$, $\mathrm{F}=5.714, p<.019)$. No significant differences were found between low and high marketing influence conditions. These findings support the inverted U-shaped relationship as proposed and clarify the causality between marketing influence and its intelligence dissemination. Marketing influence in fact causes the inverted U-shaped effect on intelligence dissemination.

Our research contributes to and extends marketing theory in several ways. Theoretically, it extends previous research on marketing influence and market orientation by providing support for an inverted U-shaped relationship between marketing influence and its intra-organizational market intelligence dissemination. From a management perspective, our findings indicate that executives have to carefully manage the influence of the marketing department in order to reach an optimal level of collaboration and sharing of market-related information within the organization. Future research could investigate boundary conditions of the inverted U-shaped relationship (e.g., organizational strategy like short vs. long-term focus) and further outcomes of influence imbalance between organizational departments. 